\title{
ESTUÁRIO DO RIO CAMBORIÚ-SC: VARIAÇÃO INTRAMAREAL E TRANSPORTE RESIDUAL DE NUTRIENTES, COP E CLOROFILA-a EM CONDIÇÕES DE QUADRATURA E SIZÍGIA
}

\author{
PEREIRA FILHO, J.*; SPILLERE, L.C.; SCHETTINI, C.A.F. \& L.F. SILVA \\ C.T.T. Mar - Universidade do Vale do Itajaí. R.Uruguai, 458 - 88.302-202 - Itajaí -SC \\ *Email: jura@cttmar.univali.br
}

\begin{abstract}
RESUMO
O objetivo deste trabalho foi avaliar a variabilidade intramareal e quantificar o transporte residual de nutrientes, Carbono Orgânico Particulado (COP) e Clorofila-a (Cla) no estuário do rio Camboriú, durante condições de maré de sizígia e quadratura. Foram realizadas 2 campanhas de amostragem de 25 horas, cobrindo dois ciclos de maré em cada. Nas campanhas foram obtidos registros de velocidade de corrente e perfis verticais de salinidade e temperatura, bem como amostras de água (superfície e fundo). A variação dos nutrientes foi maior na campanha realizada em condições de meré de sizígia. Na quadratura, o estuário ficou sempre estratificado e apresentou menor variação. Na sizígia o transporte resultou em exportação de NID, PID, Si e COP, respectivamente iguais a $-2.6 \times 10^{4},-0.9 \times 10^{3},-9.9 \times 10^{3}$ e $-1.9 \times 10^{3}$ (mol/d) e importação de Cla de $50 \mathrm{~g} / \mathrm{d}$. Na quadratura o transporte resultou em exportação de NID, PID, Si, COP e Cla de, respectivamente, $1.5 \times 10^{4},-0.35 \times 10^{3},-23 \times 10^{3},-4.4 \times 10^{3}(\mathrm{~mol} / \mathrm{d})$ e $-2.0 \times 10^{3} \mathrm{~g} / \mathrm{d}$. As maiores transferências de Si e COP na quadratura podem estar associadas ao período mais chuvoso em que ela foi realizada. $O$ menor transporte de NID e PID pode ser explicado pela sua adsorção ao material em suspensão e/ ou ao consumo pelo fitoplâncton, no interior do estuário. Esta hipótese é apoiada pela exportação de Cla nesta situação.
\end{abstract}

Palavras Chave: eutrofização, estuário, ciclo de maré, costa de SC.

\section{CAMBORIÚ RIVER ESTUARY: INTRATIDAL VARIATION AND NET TRANSPORT OF DISSOLVED NUTRIENTS, POC AND CHLOROPHYLL A DURING NEAP AND SPRING TIDE CONDITIONS}

\begin{abstract}
The purpose of this study was to evaluate the intratidal variability and to quantify the net transport of dissolved nutrients, particulate organic carbon (POC) and Chlorophyll a in the estuary of the Camboriú River during neap and spring tides. Two 25-hour continuous sampling surveys were carried out, covering two tidal cycles on each situation. On each survey, continuous records of direction and velocity of currents and vertical profiles of salinity and temperature were obtained, as well as samples of surface and botton water. The distribution of dissolved nutrients was determined by the tide during spring tide conditions and by the river discharge during neap tide conditions. In the spring tide the net transports of DIN, DIP, Si, POC and Chla-a through the estuary were $-2.6 \times 10^{4},-0.9 \times 10^{3},-9.9 \times 10^{3} \mathrm{e}-1.9 \times 10^{3}(\mathrm{~mol} / \mathrm{d})$ and $50(\mathrm{~g} / \mathrm{d})$, respectively. In the neap tide, the net transports of DIN, DIP, Si, POC and Cla-a were $-1.5 \times 10^{4},-0.35 \times 10^{3},-23 \times 10^{3},-4.4 \times 10^{3}(\mathrm{~mol} / \mathrm{d})$ e $-2.0 \times 10^{3} \mathrm{~g} / \mathrm{d}$, respectively. The largest transfers of $\mathrm{Si}$ and POC in the neap tide may be associated to the rainy period in this situation. The smallest transport of DIN and DIP can be explained by its adsorption into the particulate material and by its biological consumption. The second hypothesis is reinforced by the export of Cla-a from the estuary.
\end{abstract}

Keywords: nutrients, estuary, tide cycle, particulate organic carbon, coastal zone of SC. 


\section{INTRODUÇÃO}

A principal via de entrada de nutrientes para o ambiente marinho costeiro é a drenagem continental. A água fluvial apresenta níveis de concentração de nutrientes que superam em ordens de grandeza aqueles encontrados no mar. A alta concentração de nutrientes ocorre em função de que estes corpos d'água carreiam grande quantidade de material continental, seja de origem antrópica ou originados do intemperismo da crosta.

A região costeira apresenta produtividade primária elevada e alta riqueza biológica em função do aporte continental. O fornecimento de nutrientes é intensificado em estuários situados em regiões de elevada concentração populacional, em função do aporte de efluentes domésticos, industriais e agrícolas e do escoamento urbano. A eutrofização nas águas estuarinas e costeiras provoca diversas modificações no meio, podendo produzir um aumento da produtividade (Nixon,1992) e rendimento pesqueiro (Cederwall \& Elmgren, 1980; Nixon, 1982; Nixon et al, 1986). Entretanto a eutrofização pode ser excessiva, particularmente em ambientes de circulação restrita como baías, enseadas e lagoas costeiras, podendo causar efeitos indesejados.

Várias alterações nas características químicas e na qualidade da água destes corpos d'água também têm sido registradas, como resultado da alteração dos fluxos biogeoquímicos, com várias conseqüências ecológicas, e.g., alteração da composição de espécies (Beukema 1991), aumento de blooms fitoplanctônicos e diminuição dos níveis de oxigênio (Parker \& O'Reilly 1991, Pennock et al 1994). Diversos estudos têm sido feitos relacionando as alterações do meio em função do aporte de nutrientes e matéria orgânica (Berounsky \& Nixon 1985; Innamorati \& Giovanardi, 1990; Kimor, 1996; Carmouze \& Vasconcelos, 1992; Braga et al, 2000). No entanto, poucos quantificam tais entradas (Nixon, 1982; Nixon \& Pilson, 1984; Smith \& Veeh, 1989; Niencheski \& Windom, 1994).
Os sistemas costeiros recebem diretamente o impacto das atividades humanas ocorridas nas bacias de drenagem. Como resultado, grande parte dos compostos de origem antrópica tende a ficar concentrada nestas regiões, ao invés de se dispersar ao longo de todo o corpo oceânico (Bishop 1983, Perès et al, 1980). O tipo de influência que este aporte pode ter sobre o sistema depende dos usos e ocupação que se faz na bacia de drenagem. Zonas altamente industrializadas se caracterizam pelo aporte de metais pesados, hidrocarbonetos, nutrientes e matéria orgânica; já regiões agrícolas principalmente pela entrada de fertilizantes, além de pesticidas e herbicidas (Ribeiro, 1996).

O esgoto doméstico é outro problema diretamente relacionado à presença de centros urbanos nas proximidades de estuários. Além de grande quantidade de água, o esgoto doméstico carrega uma série de substâncias poluentes, onde se destacam a matéria orgânica, os nutrientes, organismos patogênicos e material em suspensão (Bishop, 1983). Muitos centros urbanos lançam seus esgotos, bruto ou parcialmente tratado, diretamente em águas estuarinas e costeiras. Entretanto o processo de mistura em regiões costeiras, e particularmente em estuários e lagoas costeiras, não é completo. A entrada destes efluentes em corpos d'água de circulação restrita pode provocar a eutrofização do meio, com grande desenvolvimento dos produtores primários, tanto macroalgas como fitoplâncton (Carneiro et al, 1994; Carneiro \& Barroso, 1993; Carmouze \& Vaconcelos, 1992).

Apesar da pequena dimensão do Estuário do Rio Camboriú, ele apresenta grande importância por ser a principal via de entrada de material continental para a Enseada de Camboriú, um dos principais balneários do sul do Brasil. Desta forma, a qualidade da água da enseada pode ser grandemente influenciada pelo material transportado através deste estuário. Assim, este trabalho teve como objetivo avaliar a variabilidade intramareal dos nutrientes inorgânicos dissolvidos $\left(\mathrm{NO}_{3}^{-}, \mathrm{NO}_{2}{ }^{-}, \mathrm{NH}_{4}{ }^{+}\right.$, 
$\mathrm{PO}_{4}^{3-}$ e Si), Carbono Orgânico Particulado (COP) e clorofila-a (cla) no estuário e quantificar o seu transporte para a zona costeira adjacente durante ciclos completos de maré.

\section{ÁREA DE ESTUDO}

O estuário do rio Camboriú está localizado no Sul do Brasil, no estado de Santa Catarina (figura 1). Ele representa a porção terminal do Rio Camboriú, o qual apresenta 40 $\mathrm{km}$ de extensão, drenando uma área de 200 km2. A bacia de drenagem do Rio Camboriú inclui várias regiões onde a atividade agrícola se destaca.

A caracterização hidrológica e morfológica do estuário foi feita por Schettini et al (1996) e Siegle et al (1998). A maré local é microtidal, principalmente semi-diurna, apresentando amplitude média de $0,8 \mathrm{~m}$ com valor máximo de 1,2 m. A influência meteorológica sobre a altura da maré é também importante, podendo elevá-la até cerca de $1 \mathrm{~m}$ acima da maré astronômica (Schettini et al, 1996, Carvalho et al, 1996 in Siegle et al, 1999). Segundo Siegle (1999) e Siegle et al (1999), o estuário do rio Camboriú é um estuário raso, do tipo parcialmente misturado (tipo $2 \mathrm{~b}$ segundo dia- grama de classificação de Hansen \& Rattray, 1966). Durante condições de maré de quadratura é verificado um padrão de estratificação contínuo ao longo do tempo, e durante a maré de sizígia a estratificação é observada apenas durante certos períodos do ciclo de maré.

O estuário do Rio Camboriú, nas proximidades de sua desembocadura, apresenta um canal de aproximadamente $120 \mathrm{~m}$ de largura, com cerca de $2 \mathrm{~m}$ de profundidade, sendo margeado por vegetação de manguezal, já degradada. A área encontra-se impactada, recebendo o aporte direto de esgotos domésticos e funcionando também como atracadouro para dezenas de embarcações. O estuário recebe também o efluente das lagoas de estabilização do município de Balneário Camboriú, que promovem o tratamento do esgoto bruto da cidade. A estação de tratamento consiste de duas lagoas anaeróbicas e duas lagoas facultativas, as quais recebem o esgoto de aproximadamente $85 \%$ do município. Sendo um dos mais importantes balneários do sul do Brasil, o município de Balneário Camboriú apresenta uma população fixa de aproximadamente 70 mil habitantes. Entretanto nos períodos de verão, este número se aproxima a 300 mil, o que faz com que a carga orgânica seja igualmente
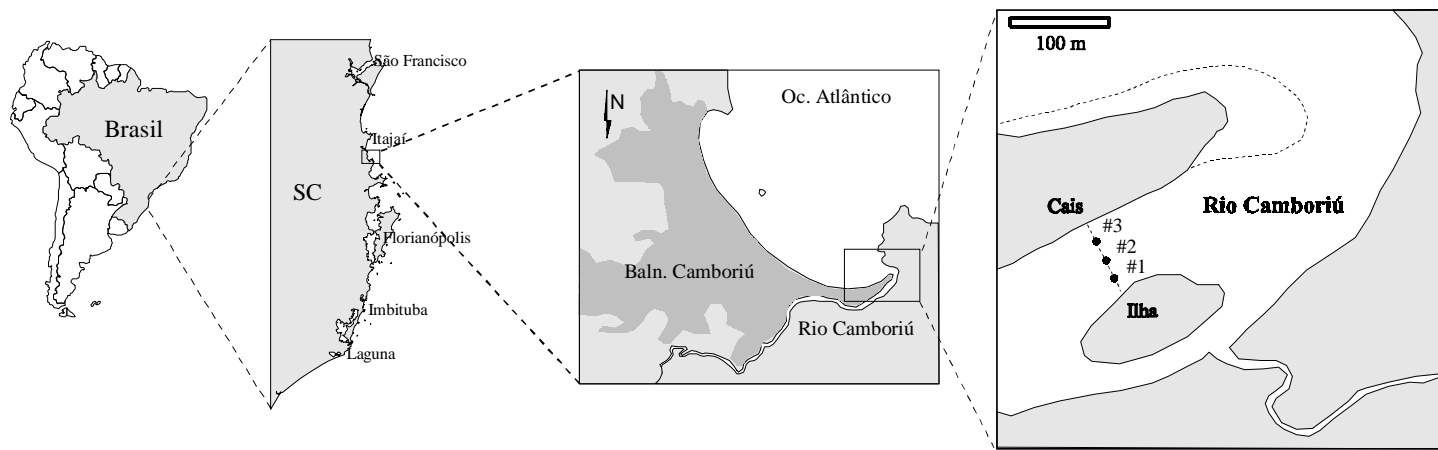

Figura 1: Localização da área de estudo com a estação de amostragens (\#2) ao longo da seção transversal (---) no estuário do rio Camboriú. 
aumentada. Desta forma, a variação sazonal da qualidade da água na enseada de Camboriú é notável, com os piores valores sendo registrados no verão, principalmente no sul da enseada, região que sofre influência direta do estuário (Kuroshima et al, 2000).

A partir do trabalho de Morelli (1997), também fica clara a importância do estuário do Rio Camboriú na influência da distribuição de nutrientes da enseada. Durante a maior parte do ano, a região sul da enseada apresenta as maiores concentrações, uma vez que está sob influência direta do estuário. As elevadas concentrações são originadas do aporte estuarino, já que este apresenta concentrações de nutrientes e COP muito maiores do que a enseada (Pereira Filho et al, 2001). Em função do aporte de nutrientes, a região sul da enseada apresenta alta produtividade primária, resultando em concentrações maiores de clorofila-a. Dependendo das correntes de maré, esta região de maior biomassa fitoplanctônica pode fluir para dentro do estuário (enchente) ou localizar-se mais externamente a ele, na enseada (vazante), como foi mostrado por Pereira Filho et al (2001).

\section{MATERIAL E MÉTODOS}

\section{Amostragem}

Para avaliar a variação temporal e estimar o fluxo de substâncias no estuário, foram realizadas duas campanhas sob diferentes condições, uma em maré de sizígia e outra em maré de quadratura. As campanhas foram realizadas próximas à desembocadura do estuário, onde a área da seção foi determinada a partir do perfil batimétrico (figura 2) realizado através de ecossonda (Siegle et al, 1999). A primeira campanha, correspondendo à maré de quadratura, foi realizada nos dias 09 e 10 de abril de 1999 e a segunda, correspondente a maré de sizígia, nos dias 27 e 28 de agosto de 1999.

Cada campanha cobriu dois ciclos completos de maré, totalizando 25 horas de amostragem em cada. Medições de velocidade e direção das correntes foram feitas em duas profundidades em uma estação fixa, no centro do canal do estuário, denominada estação 2 (figura 1), utilizando-se correntógrafos fundeados. Perfis verticais de salinidade e temperatura foram realizados a cada hora e o nível da água foi monitorado com um marégrafo de pressão fixo na margem. Amostras de água de superfície e fundo foram obtidas no momento da realização dos perfis de salinidade. As amostras foram filtradas em filtros Whatman GF/C pré-calcinados $\left(550^{\circ} \mathrm{C} / 4\right.$ horas), logo após a coleta. Os filtros foram lavados com $\mathrm{Na}_{2} \mathrm{SO}_{4}$ para eliminação de cloretos e congelados a $15^{\circ} \mathrm{C}$. O material filtrado, destinado à determinação dos nutrientes inorgânicos dissolvidos, foi então dividido em sub-amostras, congelado e posteriormente analisado em laboratório.

\section{Análise}

A partir do material retido nos filtros, foram determinados o COP e a cla. O COP foi determinado por digestão ácida com dicromato de potássio, sendo o excesso titulado com sulfato ferroso amoniacal, segundo método descrito por Grashoff et al (1983). A extração da cla foi feita com acetona $90 \%$, mediante desintegração do filtro e solvente. A determinação foi realizada por meio de cromatografia líquida de alta eficiência de fase reversa (HPLC) segundo método descrito por Mantoura et al (1997), sendo que os extratos foram clarificados antes da injeção no cromatógrafo, por filtração em filtro Whatman GF/C. Os nutrientes inorgânicos dissolvidos $\left(\mathrm{N}_{-} \mathrm{NH}_{4}{ }^{+}, \mathrm{N}^{-\mathrm{NO}_{2}}{ }^{-}, \mathrm{N}_{-} \mathrm{NO}_{3}{ }^{-}\right.$ , $\mathrm{Si}-\mathrm{H}_{4} \mathrm{SiO}_{4}$ e $\mathrm{P}-\mathrm{PO}_{4}{ }^{3-}$ ) foram determinados a partir do material filtrado, segundo métodos colorimétricos clássicos, adaptados de Strickland \& Parsons (1972). 


\section{Cálculo do Transporte}

A taxa de transporte através da seção transversal $\left(Q, \mathrm{mmol}^{-\mathrm{d}^{-1}}\right.$ ou $\left.\mathrm{mg}^{\mathrm{d}} \mathrm{d}^{-1}\right)$ de cada parâmetro em cada campanha foi obtida a partir da soma do transporte em superfície(Qs) e fundo (Qf), segundo a expressão:

$$
\begin{aligned}
& Q_{s}=\sum c_{s .} u_{s .} A_{i} / 2 \\
& Q_{f}=\sum c_{f} \cdot u_{f} \cdot A_{i} / 2 \\
& Q=Q_{s}+Q_{f}
\end{aligned}
$$

onde:

Ai é a área instantânea da seção transversal $\left(\mathrm{m}^{2}\right)$ no momento da amostragem.

c é o parâmetro de concentração $\left(\mathrm{mmol} . \mathrm{m}^{-3}\right.$ ou $\mathrm{mg} \cdot \mathrm{m}^{-3}$ ) em superfície $\left(\mathrm{c}_{\mathrm{s}}\right)$ ou fundo $\left(\mathrm{c}_{\mathrm{f}}\right)$ no momento de cada amostragem.

u é a velocidade de corrente $\left(m \cdot h^{-1}\right)$ em superfície $\left(u_{s}\right)$ ou fundo $\left(u_{f}\right)$. Por convenção, valores positivos representam correntes da região costeira para o estuário e valores negativos o inverso.
A figura 2 mostra a seção transversal no ponto de amostragem, bem como as áreas máximas e mínimas registradas nas campanhas de sizígia e de quadratura.

\section{RESULTADOS E DISCUSSÃO}

As figuras 3 e 4 e as tabelas 1 e 2 sumariam os resultados obtidos nas duas campanhas de amostragem, em situação de sizígia e de quadratura, respectivamente.

\section{Maré de Sizígia}

\section{Nutrientes Inorgânicos Dissolvidos}

A campanha feita sob condições de maré de sizígia foi realizada em agosto-99, durante um período seco. A precipitação total registrada em agosto foi de $4.8 \mathrm{~mm}$, obtida pela estação meteorológica do Centro de Ciências Tecnológicas da Terra e do Mar (CTT Mar), em Itajaí. Nesta situação a influência da maré na

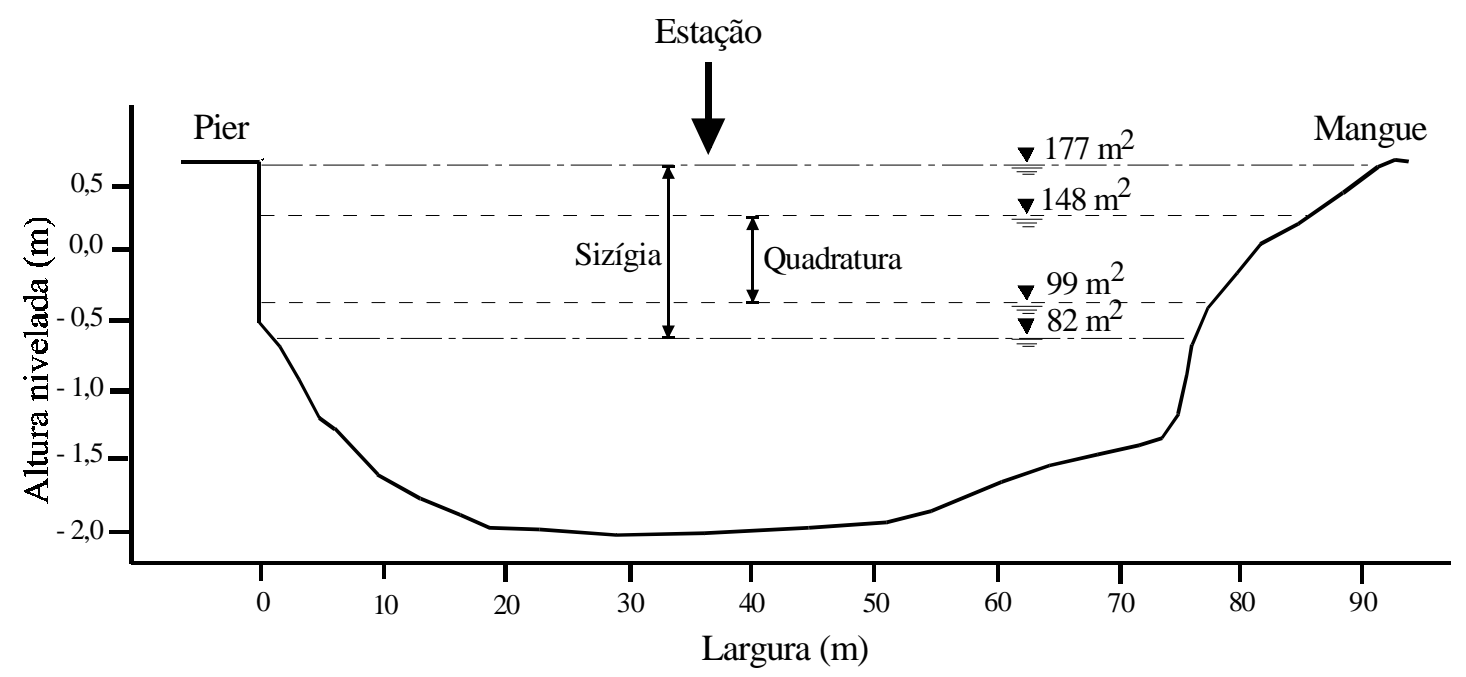

Figura 2: Seção transversal no ponto de amostragem, mostrando as áreas máximas e mínimas da seção registrados durante as amostragens, em função do nível d’água (N.A.), em condições de sizígia e quadratura. (Seção vista do estuário em direção ao oceano). 

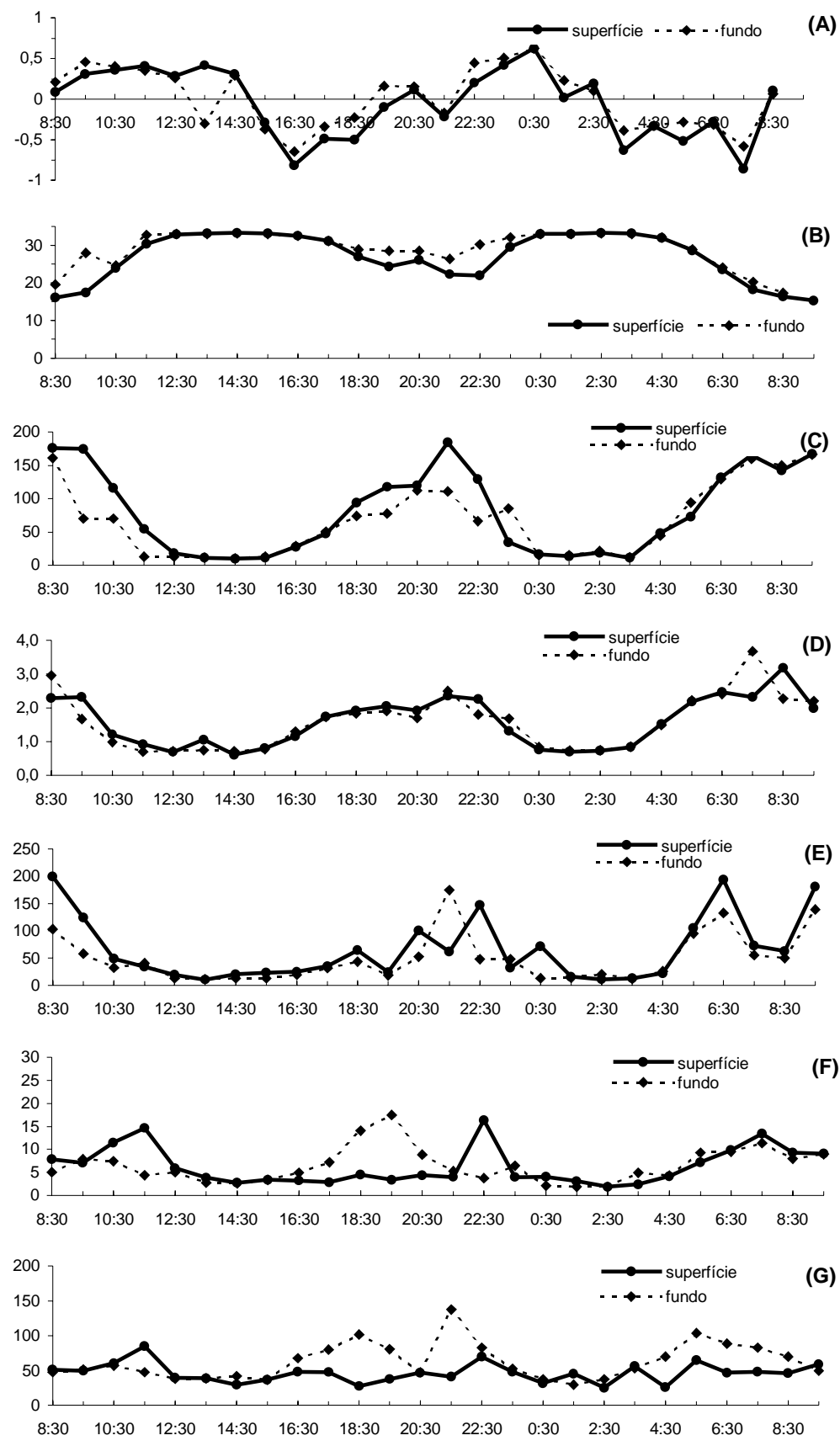

Figura 3: Variação dos parâmetros amostrados ao longo do tempo, obtidos durante condições de maré de sizígia

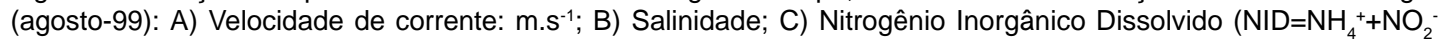
$+\mathrm{NO}_{3}{ }^{-}$): $\mathrm{mmol}^{-3} \mathrm{~m}^{-3}$ D)Fósforo Inorgânico Dissolvido: $\mathrm{mmol}^{-3}$; E) Si Inorgânico Dissolvido: $\mathrm{mmol}^{-3} \mathrm{~m}^{-3}$, F) Clorofila-a: $\left.\mathrm{mg} \cdot \mathrm{m}^{-3}, \mathrm{G}\right)$ Carbono Orgânico Particulado: $\mathrm{mmol} \cdot \mathrm{m}^{-3}$. 

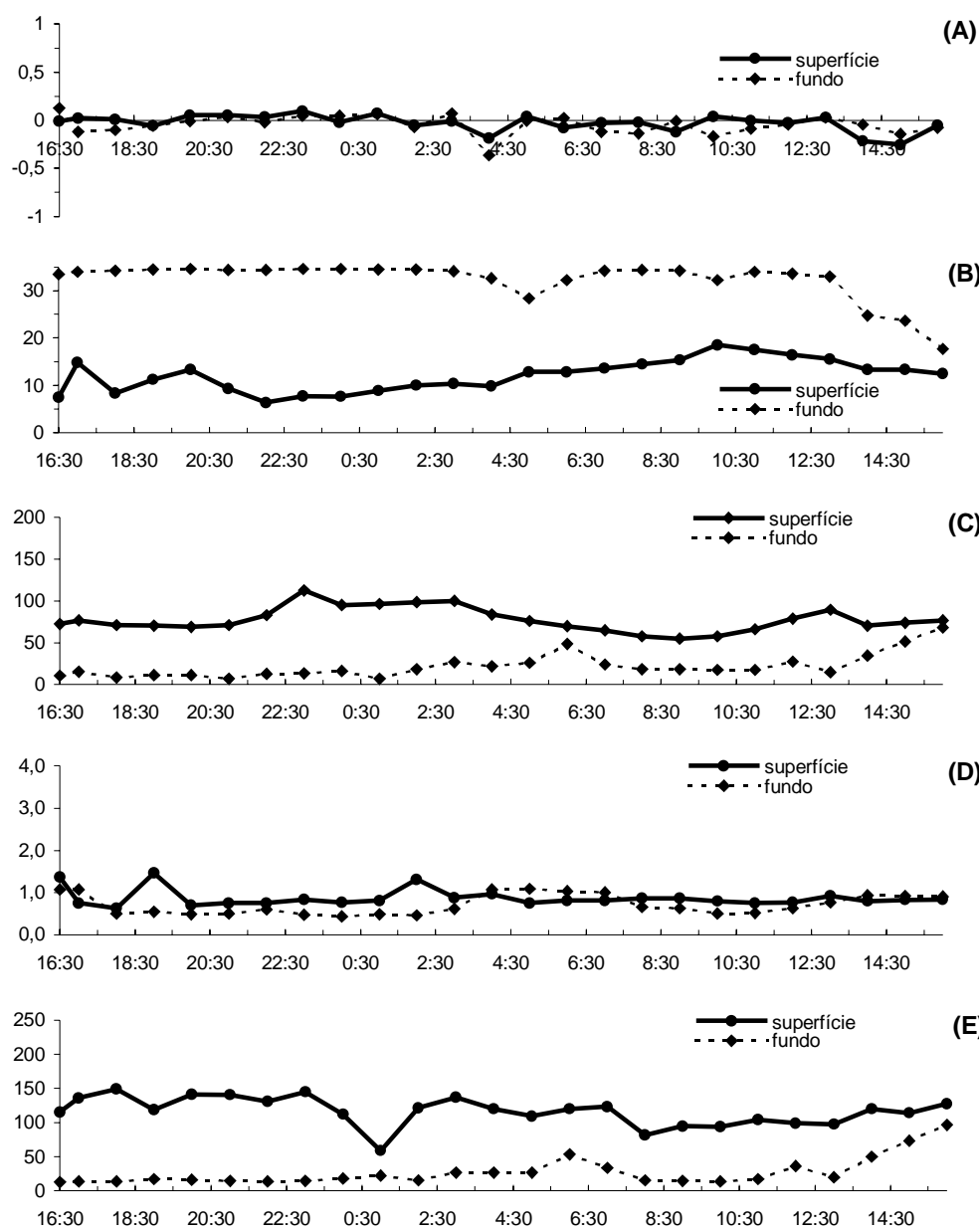

(E)

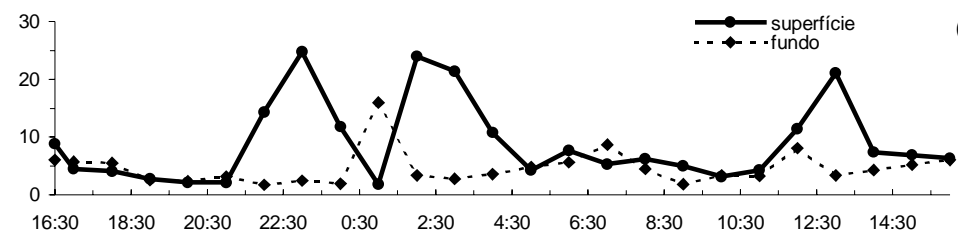

(F)

(D)

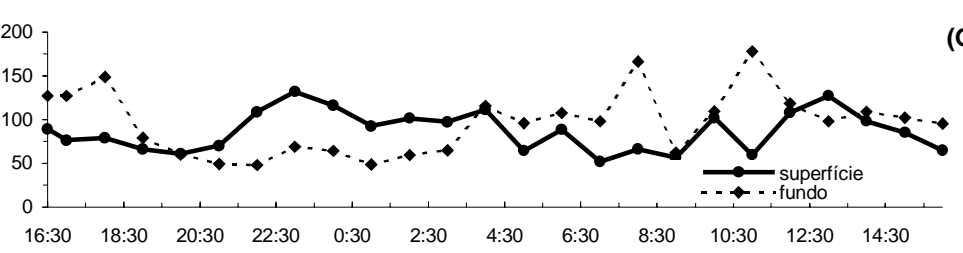

(G)

Figura 4: Variação dos parâmetros amostrados ao longo do tempo, obtidos durante condições de maré de quadratura (abril-99): A) Velocidade de corrente: $\mathrm{m}^{-\mathrm{s}^{-1}}$; B) Salinidade; C) Nitrogênio Inorgânico Dissolvido $\left(\mathrm{NID}=\mathrm{NH}_{4}{ }^{+}+\mathrm{NO}_{2}{ }^{+}+\right.$ $\mathrm{NO}_{3}{ }^{-}$): $\mathrm{mmol}^{-3}$; D)Fósforo Inorgânico Dissolvido: $\mathrm{mmol}^{-3}{ }^{-3}$ E) Si Inorgânico Dissolvido: $\mathrm{mmol}^{-3}$, F) Clorofila-a: $\left.\mathrm{mg} \cdot \mathrm{m}^{-3}, \mathrm{G}\right)$ Carbono Orgânico Particulado: $\mathrm{mmol} . \mathrm{m}^{-3}$. 
Tabela 1: Média, Desvio padrão e mínimo e máximo dos nutrientes, Clorofila e coeficientes de correlação dos mesmos com a salinidade durante a campanha realizada durante condições de maré de sizígia.

\begin{tabular}{l|lllllllllll}
\hline \hline & Salin. & $\mathrm{Temp}$ & $\mathrm{NH}_{4}^{+}$ & $\mathrm{NO}_{2}{ }^{-}$ & $\mathrm{NO}_{3}{ }^{-}$ & $\mathrm{NID}$ & $\mathrm{PO}_{4}{ }^{3-}$ & $\mathrm{N}: P$ & $\mathrm{Si}$ & $\mathrm{Cla}$ & $\mathrm{COP}$ \\
\hline Unid. & & $\left({ }^{\circ} \mathrm{C}\right)$ & $(\mu \mathrm{M})$ & $(\mu \mathrm{M})$ & $(\mu \mathrm{M})$ & $(\mu \mathrm{M})$ & $(\mu \mathrm{M})$ & & $(\mu \mathrm{M})$ & $(\mu \mathrm{g} / \mathrm{l})$ & $(\mu \mathrm{M})$ \\
& & & & & & & & & & & \\
Média & 28.9 & 18.9 & 66.3 & 1.3 & 3.4 & 71.1 & 1.5 & 39.9 & 52.6 & 6.4 & 54.7 \\
Dv. Pd & 5.0 & 0.4 & 53.8 & 1.0 & 1.6 & 56.2 & 0.7 & 22.1 & 49.1 & 4.0 & 22.7 \\
Min & 16.1 & 18.2 & 8.5 & 0.15 & 1.5 & 10.1 & 0.6 & 0.7 & 10.0 & 1.9 & 25.3 \\
Max & 33.3 & 19.9 & 168.5 & 3.5 & 7.2 & 184.3 & 3.7 & 83.8 & 199.5 & 16.3 & 137.2 \\
$\mathrm{r}$ & & -0.55 & -0.92 & -0.92 & -0.93 & -0.93 & -0.84 & -0.79 & -0.67 & -0.48 & -0.22 \\
$\mathrm{n}$ & 50 & 52 & 52 & 52 & 52 & 52 & 52 & 52 & 52 & 52 & 52 \\
\hline \hline
\end{tabular}

Tabela 2: Média, Desvio padrão, mínimo e máximo dos nutrientes, Clorofila e coeficientes de correlação dos mesmos com a salinidade durante a campanha realizada durante condições de maré de quadratura.

\begin{tabular}{l|lllllllllll}
\hline & Salin. & $\mathrm{Temp}$ & $\mathrm{NH}_{4}^{+}$ & $\mathrm{NO}_{2}{ }^{-}$ & $\mathrm{NO} 3$ & $\mathrm{NID}$ & $\mathrm{PO}_{4}{ }^{3-}$ & $\mathrm{N}: P$ & $\mathrm{Si}$ & $\mathrm{Cla}$ & $\mathrm{COP}$ \\
\hline & & $\left({ }^{\circ} \mathrm{C}\right)$ & $(\mu \mathrm{M})$ & $(\mu \mathrm{M})$ & $(\mu \mathrm{M})$ & $(\mu \mathrm{M})$ & $(\mu \mathrm{M})$ & & $(\mu \mathrm{M})$ & $(\mu \mathrm{g} / \mathrm{l})$ & $(\mu \mathrm{M})$ \\
& & & & & & & & & & & \\
Média & 22.2 & 23.0 & 45.5 & 0.9 & 3.6 & 50.0 & 0.8 & 62.4 & 72.9 & 6.7 & 90.3 \\
Dv. Pd & 10.8 & 0.5 & 29.3 & 0.5 & 2.5 & 31.6 & 0.2 & 36.2 & 50.4 & 5.9 & 30.5 \\
$\operatorname{Min}$ & 7.44 & 22.1 & 4.9 & 0.21 & n.d. & 6.87 & 0.44 & 13.6 & 12.9 & 1.71 & 47.7 \\
$\operatorname{Max}$ & 34.6 & 23.9 & 104.5 & 1.73 & 10.5 & 112.4 & 1.45 & 133.4 & 149.2 & 24.8 & 165.9 \\
$\mathrm{r}$ & & -0.66 & -0.94 & -0.84 & -0.69 & -0.95 & 0,19 & -0.89 & -0.94 & -0.41 & -0.10 \\
$\mathrm{n}$ & 50 & 50 & 50 & 50 & 50 & 50 & 50 & 50 & 50 & 50 & 50 \\
\hline \hline
\end{tabular}

dinâmica do estuário é mais perceptível. Durante a maré alta, com a entrada de água salgada, a qual é observada pelos valores de velocidade de corrente positivos (figura $3 \mathrm{~A}$ ), verificou-se aumento da salinidade (figura 3B) e homogeneização da coluna. A salinidade nesta campanha variou entre 16.1 e 33.3, como pode ser visto na figura $3 \mathrm{~B}$. A variação da salinidade refletiu a influência da maré no estuário. Assim, no início da campanha, durante um período de entrada de água (corrente positiva, figura $3 \mathrm{~A}$ ), observa-se aumento da salinidade. Este aumento é observado até as 11:30 hs, quando as salinidades de superfície e fundo se igualam em torno de 32. Após um período de coluna d'água homogênea, registrado entre as 12:30 e 17:30 hs, observa-se diminuição da salinidade. Esta diminuição ocorreu até a 22:30 hs, quando a salinidade ficou novamente em torno de 16.0. Esta diminuição ocorreu após um período de vazante, verificada pela velocidade de corrente negativa, entre 15:30 e 20:30 hs (figura 3 A). No ciclo de maré seguinte verificou-se o mesmo padrão.

A variação da maré, que provocou entrada de água salgada no estuário, se torna evidente na evolução das concentrações de nutrientes. As maiores concentrações de nutrientes de NID, $\mathrm{PO}_{4}{ }^{3-}$ e Si coincidiram com os períodos de maré baixa, em intervalos de aproximadamente 13horas(figuras 3C, 3D, 3E, respectivamente). Assim, no início da amostragem, durante um período de salinidade em torno de 16 , foram registrados valores ele- 
vados de todos os nutrientes. Com a elevação da salinidade, observada até as 12:30hs, as concentrações dos nutrientes diminuem, permanecendo baixas até as 14:30 hs. Com a inversão das correntes (figura 3 A), evidenciando o início da vazante, verifica-se diminuição da salinidade (figura 3 B) e aumento de nutrientes até um novo pico, registrado as $22: 30 \mathrm{hs}$. No ciclo de maré seguinte, observa-se um padrão de variação semelhante. Este intervalo de aproximadamente 13 horas entre os picos de nutrientes reflete a grande influência da maré nesta situação. Os maiores valores de nutrientes estão associados a períodos de menor salinidade, durante a maré baixa, com grande contribuição de águas fluviais (figura 3B). Como resultado, verificou-se uma elevada correlação inversa entre salinidade e concentração de nutrientes (tabela 1).

As correlações negativas com a salinidade foram marcantes para os nutrientes nitrogenados dissolvidos $\left(\mathrm{NID}=\mathrm{NH}_{4}{ }^{+}+\mathrm{NO}_{2}{ }^{-}\right.$ $\mathrm{NO}_{3}{ }^{-}$). O NID é freqüentemente mencionado como limitante à produção primária na zona costeira (Rimmelin et al, 1998). Por outro lado o seu aumento está freqüentemente relacionado ao grau de poluição doméstica e agropecuária de um ambiente aquático (Carmouze, 1994). No caso do Estuário do Rio Camboriú, foram sempre observados valores elevados de NID, particularmente na água mais doce. Estes valores tão altos ocorreram em função da grande quantidade $\mathrm{NH}_{4}{ }^{+}$no meio. $\mathrm{O}$ $\mathrm{NH}_{4}{ }^{+}$foi responsável por aproximadamente $90 \%$ do NID, chegando a atingir valores de até 169 $\mathrm{mM}$. Estes valores são extremamente altos para águas naturais. Mesmo em outros ambientes degradados do litoral brasileiro, as concentrações de $\mathrm{NH}_{4}{ }^{+}$dificilmente atingem este valor. É o caso do sistema estuarino da Baixada Santista, no litoral sul de São Paulo, onde as concentrações máximas de $\mathrm{NH}_{4}{ }^{+}$durante 0 verão não ultrapassam $100 \mathrm{mM} \mathrm{N}-\mathrm{NH}_{4}{ }^{+}$(Braga et al, 2000). O estuário do Rio Itajaí, situado a cerca de $10 \mathrm{~km}$ ao norte do estuário do Camboriú também apresenta concentrações máximas de $\mathrm{NH}_{4}{ }^{+}$de até $60 \mathrm{mM}$ (Spillere, 2002), inferiores às máximas encontradas neste estudo. A alta concentração de $\mathrm{NH}_{4}{ }^{+}$está provavelmente associada ao lançamento do efluente da estação de tratamento de esgotos do município de Balneário Camboriú. A estação, situada aproximadamente $1.5 \mathrm{~km}$ a montante do ponto amostrado, recebe e trata o esgoto de cerca de $85 \%$ do município (Kuroshima et al, 2000). Além disso, o rio recebe o lançamento de esgotos do município de Camboriú, que não dispõe de sistema de tratamento (Kuroshima et al, 2000). Estas entradas representam provavelmente a origem do aporte excessivo de $\mathrm{NH}_{4}{ }^{+}$no sistema, o que vem alterando a qualidade da água na enseada de Camboriú, como tem sido observado em outros trabalhos (Kuroshima, 2000; Morelli, 1997).

Apesar das altas concentrações observadas para o $\mathrm{NH}_{4}^{+}$, o $\mathrm{PO}_{4}^{3-}$ e Si não mostraram concentrações tão elevadas. $\mathrm{O} \mathrm{PO}_{4}{ }^{3-}$ variou entre 0.6 e $3.5 \mathrm{mM}$ de $\mathrm{P}_{-} \mathrm{PO}_{4}{ }^{3-}$, com os maiores valores registrados nos períodos de menor salinidade, seguindo o ciclo de maré (figura 3D). Já o Si variou entre 10.0 e 199.5 mM Si$\mathrm{H}_{4} \mathrm{SiO}_{4}$, também com os maiores valores registrados em períodos de maré baixa, com menor salinidade (figura 3E).

A partir dos valores de NID e PID, foi calculada a razão molar N:P que apresentou média para todo o período de aproximadamente $40: 1$, com picos superiores a 100 em períodos de baixa salinidade. Esta alta razão, se comparada a razão de Redfield (NID:PID 16:1; Redfield, 1958), poderia ser ocasionada por uma alta concentração de NID e/ou por uma baixa concentração de $\mathrm{PO}_{4}^{3-}$. Várias evidências têm demonstrado que os estuários funcionam como locais de remoção geoquímica de $\mathrm{PO}_{4}{ }^{3-}$, como resultado da adsorção e floculação (Day et al, 1989). Neste estudo entretanto, os dados são insuficientes para que esta hipótese seja comprovada, uma vez que o $\mathrm{PO}_{4}^{3-}$ demonstrou uma elevada correlação negativa com a salinidade (tabela 1), o que sugere um comportamento conservativo.

A comparação da razão molar N:P encontrada neste estudo com a de Redfield, 
embora seja uma aproximação grosseira para esta situação, já que o ambiente é estuarino e impactado, indica excesso de NID no ambiente. De fato os valores encontrados correspondem à grande quantidade de $\mathrm{NH}_{4}^{+}, \mathrm{O}$ que pode estar associado à decomposição de matéria orgânica e à entrada antrópica. Os valores máximos de $\mathrm{NH}_{4}^{+}$encontrados no estuário são comparáveis a outros ambientes reconhecidamente impactados, como os estuários de Santos e SãoVicente, no litoral sul de São Paulo (Braga et al, 2000).

\section{Clorofila e COP}

A cla apresentou valores relativamente altos, com concentrações de até $20 \mathrm{mg} \cdot \mathrm{m}^{-3}$, com os picos associados ao ciclo de maré. Estas concentrações de cla ocorreram em função da grande entrada de nutrientes através do estuário, o que estimula o desenvolvimento fitoplanctônico. Estes organismos se desenvolvem provavelmente no encontro das águas estuarinas, ricas em nutrientes, com as águas da enseada que apresentam maior transparência, resultando na formação de um cinturão de alta produtividade próxima a desembocadura do estuário(Mann \& Lazier, 1991). Este cinturão, já relatado por Pereira Filho et al (2001), se deslocaria para dentro ou para fora do estuário, o que explicaria os picos de clorofila em intervalos aproximadamente regulares.

Os maiores valores de COP estiveram associados ao fundo, como pode ser observado pela figura 3G. Esta tendência pode ser conseqüência do efeito das correntes de maré no estuário, o que faz com que ocorra ressuspensão de material sedimentado, aumentando as concentrações no fundo.

\section{Maré de Quadratura}

\section{Nutrientes Inorgânicos Dissolvidos}

A campanha de quadratura foi realizada em abril-99, um mês que apresentou um total de precipitação equivalente a $130 \mathrm{~mm}$. Apesar disso, durante a campanha a velocidade de corrente não variou muito, oscilando sempre em torno de zero (figura 4A). Esta baixa velocidade ocorreu em função da baixa amplitude de maré, característica da condição de quadratura. Assim, em nenhum momento o estuário foi dominado apenas pela água salgada, como ocorreu na campanha de sizígia, durante a maré alta.

O que foi observado foi a presença constante de água salgada no fundo e uma água mais salobra, resultado da mistura da água fluvial com a água da enseada mais salgada, na superfície. Esta tendência é mostrada pela estratificação da coluna d'água com grande diferença de salinidade, como é mostrada na figura 4B. Este padrão, que já foi verificado nesta situação de maré em estudos anteriores (Siegle et al, 1999), e foi discutido mais detalhadamente por Silva (1999), é refletido também na falta de padrão de variação dos nutrientes. Assim, a maioria dos nutrientes apresentou concentrações altas na superfície e menores no fundo, ao longo de todo o ciclo de maré. Desta forma a variação das concentrações dos nutrientes foi menor do que na situação de sizígia, refletindo a menor amplitude da maré nesta condição (tabelas 1 e 2).

As concentrações dos nutrientes foram, em média, menores na maré de quadratura (tabelas 1 e 2). Esta diferença pode estar relacionada à presença constante de água da enseada, mais salgada, no fundo. Os nutrientes tenderam a ter concentrações maiores na superfície, devido ao predomínio da água mais doce proveniente do rio. Devido à estratificação ser praticamente constante, os nutrientes apresentaram pouca variação tanto na superfície como no fundo (figuras 4B, 4C, 4D e 4E).

Como ocorreu na maré de sizígia, o NID apresentou os maiores valores relacionados à água mais doce e sua principal forma novamente foi o amônio, representando em média $90 \%$ do NID. Para o $\mathrm{NO}_{3}^{-}$e o $\mathrm{NO}_{2}^{-}$também verificou-se que as maiores concentrações estavam relacionadas as águas de menor salinidade. 
$\mathrm{O} \mathrm{PO}_{4}^{3-}$ apresentou uma concentração mais baixa que a observada na sizígia. Esta menor concentração pode estar associada à mistura da água fluvial e marinha na superfície, dentro do estuário. A menor amplitude de maré na quadratura acaba resultando em baixa velocidade de corrente no estuário, aumentando o tempo de residência da água no mesmo. Isto provavelmente possibilita a adsorção do fosfato ao material em suspensão, diminuindo sua concentração na água. Esta diminuição é particularmente evidente na água com maior influência fluvial (superfície), fazendo com que a Razão N:P se torne elevada na superfície. O processo de floculação no estuário representa também outra forma de retirada de PID da água, aumentando os sítios adsortivos para o $\mathrm{PO}_{4}{ }^{3-}$ (Day et al, 1989).

O Si mostrou um comportamento semeIhante aos nutrientes nitrogenados, com concentrações maiores na superfície, devido ao predomínio de água doce, e menores no fundo (figura 4E).

\section{Clorofila e COP}

A cla apresentou grande variação, mostrando períodos de elevação de sua concentração, alternados por períodos de baixa concentração. Assim, as concentrações de cla mostraram-se particularmente elevadas entre as 22:00 e 0:00hs e entre as 02:00 e 03:00hs, no primeiro ciclo de maré. Estas maiores concentrações ocorrem provavelmente, em função do aumento do desenvolvimento fitoplanctônico que ocorre durante a mistura de água doce, rica em nutrientes, com a água da enseada, mais transparente (Mann \& Lazier, 1991). Nesta zona de mistura, os organismos podem se desenvolver, aproveitando os dois fatores que vão determinar o seu sucesso: luz e nutrientes. Neste estudo o grande aumento da clorofila, mostra a passagem intermitente de uma água com grandes concentrações de organismos, a qual acompanha a oscilação das correntes no estuário. A origem destes organismos é que não está bem esclarecida. Ela pode ter se formado no interior do estuário, nas proximidades da desembocadura do efluente da ETE de Balneário Camboriú (formada por organismos relacionados a águas mais doces) ou na parte mais externa, já na pluma do Rio Camboriú, (formada por organismos de origem marinha, como foi encontrado em outra situação, durante a sizígia) (Pereira Filho et al, 2001). Como não foi feita uma análise microscópica sobre a composição fitoplanctônica, esta questão fica aberta.

Os picos de Cla não foram acompanhados de aumentos de COP. Isto mostra que a maior parte do COP no estuário é de origem detrítica, não sendo relacionado diretamente com a biomassa fitoplanctônica. Este material detrítico pode ter origem tanto a partir do Rio Camboriú, como pela ressuspensão do sedimento de fundo, como já foi sugerido por Siegle (1999).

\section{Transporte}

A partir da velocidade de corrente, área da seção transversal e concentrações dos parâmetros analisados, foi calculado o transporte de material nas duas situações amostradas: sizígia e quadratura. Os resultados, mostrados na tabela 3, indicaram que com exceção da Cla, na sizígia, ocorreu uma exportação de substâncias para a enseada nas situações amostradas.

Comparando as duas situações, podese notar uma diferença nos padrões de transporte para cada substância. Assim, na maré de sizígia o transporte de NID e PID, foi maior que na quadratura. Por outro lado o transporte de Si e COP foi maior na quadratura. Estas diferenças podem estar relacionadas à origem do material, associadas aos processos de remoção dos mesmos no estuário, em cada uma das situações. Também a precipitação pode ser importante na determinação destas diferenças.

A campanha de sizígia foi realizada em agosto de 1999, durante um período relativamente seco, com precipitação total, registrada 
Tabela 3: Transporte total referente às campanhas durante os dois ciclos de maré amostrados: quadratura e sizígia. Os valores negativos representam transporte do estuário em direção a enseada.

\begin{tabular}{l|cc}
\hline Transporte total & Sizígia & Quadratura \\
\hline NID $\left(\mathrm{mol}^{-1} \mathrm{~d}^{-1}\right)$ & $-2,6 \times 10^{4}$ & $-1,5 \times 10^{4}$ \\
PID $\left(\mathrm{mol}^{-1} \mathrm{~d}^{-1}\right)$ & $-0,9 \times 10^{3}$ & $-0,35 \times 10^{3}$ \\
Si $\left(\mathrm{mol} . \mathrm{d}^{-1}\right)$ & $-9,9 \times 10^{3}$ & $-23 \times 10^{3}$ \\
Cla-a $\left(\mathrm{g} \cdot \mathrm{d}^{-1}\right)$ & 50 & $-2,0 \times 10^{3}$ \\
COP $\left(\mathrm{mol}^{-1} \mathrm{~d}^{-1}\right)$ & $-1,9 \times 10^{3}$ & $-4,4 \times 10^{3}$ \\
\hline
\end{tabular}

na estação meteorológica do CTT Mar/UNIVALI, em Itajaí, de $4.8 \mathrm{~mm}$. Nesta situação, com um menor escoamento superficial na bacia de drenagem, foi verificado um menor transporte de Si e COP. Por outro lado, a campanha de quadratura foi realizada em abril de 1999, período com maior precipitação $(130 \mathrm{~mm})$, o que explicaria estes maiores valores de transporte de Si e COP nesta situação com relação à sizígia.

Apesar das diferenças na intensidade de chuvas, o transporte de $\mathrm{NID}$ e $\mathrm{PO}_{4}{ }^{3-}$ foi maior durante a maré de sizígia. Dois possíveis processos podem explicar esta discrepância, ambos relacionados à remoção destes nutrientes na campanha de quadratura. Nesta condição, em função da menor amplitude da maré, foram registrados os menores valores instantâneos de velocidade de corrente (figura 4A). Desta forma, o material dissolvido atravessa 0 estuário mais lentamente, o que proporciona a sua adsorção ao material em suspensão. Os nutrientes que reconhecidamente tendem a sofrer adsorção são o $\mathrm{NH}_{4}^{+}$, que representou mais de $90 \%$ do NID, e, principalmente, o $\mathrm{PO}_{4}$ (Day et al, 1989). Neste caso a adsorção seria um mecanismo geoquímico que explicaria esta discrepância.

O outro processo que poderia também explicar o menor transporte de NID e $\mathrm{PO}_{4}{ }^{3-}$ na quadratura seria o consumo biológico destes nutrientes no interior do estuário, o que seria favorecido também pela sua passagem lenta através do mesmo. O transporte de clorofila, que na quadratura correspondeu a uma grande exportação para a enseada, apóia esta hipótese. Em resumo, os valores de transporte de NID e PID foram menores nesta situação em função de sua maior reatividade geoquímica e biológica.

No caso da sizígia, verificou-se uma pequena importação de clorofila-a em direção ao estuário. Nesta situação, os organismos podem ter se desenvolvido próximos à desembocadura do estuário, na enseada, no encontro das águas estuarinas ricas em nutrientes com a água da enseada, mais transparente. Esta zona de grande desenvolvimento fitoplanctônico se deslocaria para dentro (ou para fora) do estuário, dependendo da direção da maré, formando um cinturão de alta produtividade, como já foi sugerido por Pereira Filho et al (2001), nesta condição de maré.

\section{CONCLUSÃO}

O estuário do rio Camboriú apresenta uma elevada concentração de nutrientes, mostrando sinais nítidos de eutrofização, quando comparados a outros ambientes impactados, como o estuário do Rio Itajaí e o estuário da Baixada Santista em São Paulo (Spillere, 2002; Braga et al, 2000).

A variação dos nutrientes ao longo das diferentes condições de maré mostrou dois padrões distintos de variação. Na maré de sizígia a variação dos nutrientes foi maior, com períodos onde toda a coluna d'água foi dominada pela água marinha. Já na quadratura, o estuário permaneceu estratificado durante todo o ciclo de maré e a variação total dos nutrientes foi menor.

O transporte total nas duas situações de maré apresentou uma tendência a exportação, com exceção da clorofila na sizígia. Entretanto ocorreram diferenças nas taxas de transporte, que podem estar associadas aos processos de remoção, geoquímico ou biológi$\mathrm{co}$, de alguns nutrientes, dentro do estuário, como $\mathrm{NH}_{4}{ }^{+}$e $\mathrm{PO}_{4}{ }^{3-}$. Esta remoção aparentemente foi mais importante na situação de 
quadratura, em função da menor velocidade de corrente, aumentando o tempo de residência no estuário.

\section{AGRADECIMENTOS}

Os autores agradecem a Márcio da Silva pelo auxílio nas determinações de clorofilaa através de HPLC. Agradecem também à Proppex/Univali pelo suporte financeiro ao trabalho, através de uma bolsa de Iniciação Científica, Probic, e a recursos do FAP. Aos alunos do curso de Oceanografia que participaram das campanhas de amostragem: Marilu Gonzaga, Carla Abreu D’aquino, Anatole Trochimckzuc Filho, Janine Pereira e Mário Preto. Um agradecimento especial à Da lole Schettini e Eliane Trucollo pelo auxílio durante as campanhas.

\section{REFERÊNCIAS BIBLIOGRÁFICAS}

Beukema, J.J. 1991. Changes in Composition of Bottom Fauna of a Tidal-Flat Area During a Period of Eutrophication. Marine Biology, 111:293-301.

Berounsky, V.M. \& S.W. Nixon. 1985. Eutrophication and the Rate of Net Nitrification in a Coastal Marine Ecosystem. Estuarine, Coastal and Shelf Science, 20: 773-781.

Bishop, P.L. 1983. Marine Pollution and its Control. McGraw-Hill.358p.

Braga, E.S., Bonetti, C.V.D., Burone, L. \& J. Bonetti Filho. 2000. Eutrophication and Bacterial Pollution Caused by Industrial and Domestic Wastes at the Baixada Santista Estuarine System - Brazil. Marine Pollution Bulletin, 40(2):165-173.

Carmouze, J-P. 1994. O Metabolismo dos Ecossistemas Aquáticos. Editora Edgard Blücher Ltda. 254p.

Carmouze, J-P. \& P. Vasconcelos. 1992. The Eutrophication of the Lagoon of Saquarema, Brazil. The Science of the Total Environment, Suppl: 851-859.
Carneiro, M.E.R., Azevedo, C., Ramalho, N.E. \& B.A. Knoppers. 1994. A Biomassa de Chara hornemannii em Relação ao Comportamento Físico-Químico da Lagoa de Piratininga (RJ). An. Acad. bras. Ci, 66 (2):213-222.

Carneiro, M.E.R. \& L.V. Barroso. 1993. Um Gradiente de Perturbação Antrópica em dois Sistemas Lagunares do Leste Fluminense, RJ, Brasil. In: II Congresso de Geoquímica dos Países de Língua Portuguesa. Resumos: 531-534.

Cederwall, H. \& R. Elmgren. 1980. Biomass Increase of Benthic Macrofauna Demonstrates Eutrophication of the Baltic Sea.. Ophelia, Suppl 1:287-304.

Day, J.D.; Hall, C.A.S.; Kemp, W.M. \& A. Yanes-Arancibia. 1989. Estuarine Ecology. John Wiley \& Sons Ed. 558 pp.

Grasshoff, K; Ehrhardt, M. \& K. Kremling. 1983. Methods of Seawater Analysis. Verlag Chemie. 419 p.

Hansen, D.V. \& M. Rattray. 1966 New dimensions on estuarine classification. Limnology and Oceanography, 11:319-326.

Innamorati, M. \& F. Giovanardi. 1990. Interrelationships Between Phytoplankton Biomass and Nutrients in the Eutrophicated Areas of the North-Western Adriatic Sea. In: Vollenweider, R.A., Marchetti, R. \& Viviani, R. (ed). Marine Coastal Eutrophication. Proceedings. Elsevier: 235250.

Kimor, B. 1996. The Impact of Eutrophication on Phytoplankton Composition in Coastal Marine Ecosystems. In: Vollenweider, R.A., Marchetti, R. \& Viviani, R. (ed). Marine Coastal Eutrophication. Proceedings. Elsevier: 871-878.

Kuroshima, K.; Barreiros, M.A.B.; Rörig, L.R.; Scherer, P.F.; Ternes, C.F.; Fasanaro; R.; Schroeder, R.A. \& L. Ribeiro. 2000. Avaliação das Características Físicas e Químicas da Enseada de Camboriú-SC-Brasil. In: XXVII Congresso Interamericano de Engenharia Sanitária e Ambiental. (C.D.) 9 pp. 
Mann, K.H. \& J.R.N. Lazier. 1991. Dynamics of marine ecosystems. Boston. Blackwell Scientific Publications. 466p.

Mantoura, R.F.C., Barlow, R.G. \& E.J.H. Head. 1997. Simple Isocratic HPLC Methods for Chlorophylls and their Degradation Products. In: Jeffrey, SW, Mantoura, R.F.C. \& Wright, S.W. (eds). Phytoplankton Pigments in Oceanography. Unesco, p.307226.

Morelli, F. 1997. Dinâmica de Nutrientes Inorgânicos na Enseada de Camboriú, Balneário Camboriú-SC. Monografia de Conclusão do Curso de Oceanografia. Univali. 82p.

Niencheski, L.F. \& H.L. Windom. 1994. Nutrient Flux and Budget in Patos Lagoon Estuary. The Science of Total Environment, 149: 5360.

Nixon, S.W. 1982. Nutrient Dynamics, Primary Production and Fisheries Yields of Lagoons. Oceanologica Acta. Suppl.: 357-371.

Nixon, S.W. 1992. Quantifying the Relationship Between Nitrogen Input and the Productivity of Marine Ecosystems. Proceedings of the advanced Marine Technical Conference, 5: 57-83.

Nixon, S.W.; Oviatt, C.A.; Frithsen, J. \& B. Sullivan. 1986. Nutrients and the Producitivity of Estuarine and Coastal Marine Ecosystems. Journal of Limnology Society of South Africa, 12: 43-71.

Nixon, S.W. \& M.E. Pilson. 1984. Estuarine Total System Metabolism and Organic Exchange Calculated from Nutrient Ratios: An Example from Narragansett Bay. In: Kennedy, V.S. (Ed.), The Estuary As a Filter - Seventh Biennial International Estuarine Research Conference. Academic Press.

Parker, C.A. \& J.E. O'Reilly. 1991. Oxygen Depletion in Long Island Sound: a Historical Perspective. Estuaries, 14: 248-265.

Pennock, J.R.; Sharp, J.H. \& W.W. Schroeder. 1994. What Controls the Expression of Estuarine Eutrophication? Case Studies of Nutrient Enrichment in the Delaware Bay and Mobile Bay Estuaries, USA. In: Dyer,
K.R. \& Orth, R.J. (Eds.), Changes in fluxes in estuaries: Implications frem science to management. Olsen \& Olsen.

Pereira Filho, J.; Schettini, C.A.F.; Rörig, L.R. \& E. Siegle. 2001. Intratidal variation and net transport of dissolved inorganic nutrients, POC and chlorophyll-a in the Camboriú River Estuary, Brazil. Estuarine, Coastal and Shelf Science 53:249-257.

Perès, J-M; Bellan, G.; Ramade, F.; Ancellin, J.; Lourde, Ple.; Michel, P.; Gauthier, M.; Soudan, F.; Bellan-Santini, D. \& M. Fontaine. 1980. La Polución de las Aguas Marinas. Barcelona. Ed. Omega. 250 pg.

Redfield, A.C. 1958. The Biological Control of Chemical Factors in the Environment. Limnol.Oceanogr: 46: 205-221.

Ribeiro, C.H.A. 1996. Uma Análise da Qualidade de Água da Baía de Guanabara entre 1980 e 1993. Dissertação de Mestrado, Universidade Federal Fluminense, Depto de Geoquímica.

Rimmelin, P.; Dumon, J.; Maneaux, E. \& E. Gonçalves. 1998. Study of Annual and Seasonal Dissolved Inorganic Nitrogen Inputs into the Arcachon Lagoon, Atlantic Coast (France). Estuarine Coastal and Shelf Sience, 47: 649-659.

Schettini, C.A.F.; Carvalho, J.L.B. \& P. Jabor. 1996. Comparative Hydrology and Suspended Matter Distribution of Four Estuaries in Santa Catarina State Southern Brazil. In: Workshop on Comparative Studies of Temperate Coast Estuaries. Proceedings, 29-32.

Siegle, E. 1999. Morfodinâmica da Desembocadura do Rio Camboriú, Balneário Camboriú, SC. (1999). Dissertação de Mestrado. IG/UFRGS.

Siegle, E.; Klein, A.H.F.; Toldo Jr., E.E. \& J.G. Abreu. 1998. Morphology and Stability of the Camboriú River Inlet, Santa Catarina, Brazil. Journal of Coastal Research, SI (26): 94-100.

Siegle, E.; Schettini, C.A.F.; Klein, A.H.F. \& E.E. Toldo Jr. 1999. Suspended Sediment Transport and Hydrodynamics at Camboriú 
River Inlet, Brazil. Coastal Sediments'99 (ASCE). In press.

Silva, L.F. 1999. Avaliação da Dinâmica Lateral e dos Mecanismos de Transporte no Estuário do Rio Camboriú. Monografia de Conclusão de Curso. Universidade do Vale do Itajaí.

Smith, S.V. \& H.H. Veeh. 1989. Mass Balance of Biogeochemically Active Materials $(C, N, P)$ in a Hypersaline Gulf. Estuarine, Coastal and Shelf Science, 29: 195-215.
Spillere, L.C. 2001. Determinação de um Balanço de Massa e da Variabilidade dos Nutrientes Inorgânicos Dissolvidos no Estuário do Rio Itajaí-açu. Monografia de Conclusão de Curso. Universidade do Vale do Itajaí.

Strickland, J.D. \& T.R. Parsons. 1972. A Pratical Handbook of Seawater Analysis. Fisheries Research. Board of Canada Bulletin. 2nd Edition. 